\title{
Dimensional stability of a novel polyvinyl siloxane impression technique
}

\author{
Moira Pedroso Leão, Camila Paloma Pinto, Ana Paula Sponchiado, Bárbara Pick Ornaghi ${ }^{1}$ \\ Universidade Positivo - UP, School of Dentistry, Area of Prosthodontics, Curitiba, PR, Brazil
}

\begin{abstract}
Aim: To introduce a modification of the reline impression technique (MRIT), and compare the dimensional changes of impressions obtained by MRIT and by conventional reline impression technique (CRIT). Methods: An acrylic resin tablet was milled by a CAD-CAM system to simulate three abutments ( $A, B$ and $C$ ) with different distances among them. The abutments were molded using both impression techniques. For MRIT, before completing the putty silicone polymerization, the relieve procedure was made by compression and it was immediately repositioned to complete the polymerization. Impressions were stored dry at room temperature for different periods (immediately, $1 \mathrm{~h}, 2$ days and 7 days). The distances were obtained by scanning. The differences between the impressions and their respective matrix reference measurements were calculated to determine the dimensional changes. Data were subjected to ANOVA and Tukey's test $(\mathrm{p}<0.05)$. Results: For $A B$ and $B C$ distances, there was no statistically significant difference between CRIT and MRIT ( $p=0.0597$ and $p=0.2167$, respectively). For $A C$, there was statistically significant difference between the techniques for the immediate storage time $(\mathrm{p}=0.006)$. In general, for CRIT the material showed expansion, while for MRIT it showed contraction. Conclusions: It was verified that the addition silicon impressions obtained by both impression techniques showed dimensional stability, except for the immediate time-point.
\end{abstract}

Keywords: dimensional measurement accuracy; dental impression materials; dental impression technique; laboratory research.

\section{Introduction}

In the process of dental prosthesis production, impression making plays a key role, because it transfers the clinical situation to a cast, which must reproduce accurately the oral structures and simulate the occlusion with its antagonist ${ }^{1-3}$. A precise reproduction of dental structures provides prostheses with good marginal quality. The different characteristics of commercially available elastomeric impression materials influence the accuracy and the dimensional stability of the impressions ${ }^{1-4}$.

According to the American Dental Association (ADA) specification \#19, elastomers are polymeric impression materials, classified into four categories by

Received for publication: April 08, 2014 Accepted: June 05, 2014

Correspondence to: Bárbara Pick Ornaghi Rua Prof. Pedro Viriato Parigot de Souza, 5300 CEP: 81280-330, Curitiba, PR, Brasil Phone: +55 $4133173000,+554199796310$ E-mail: bpo@up.com.br, barbara@pick.com.br their chemical composition and polymerization reaction: condensation-cured silicones, addition-cured silicones, polysulfides (mercaptans) and polyethers ${ }^{2-5}$.

Ideally, the dimensional stability of an impression material reflects its ability to maintain the accuracy of the impression over time which, according to specification \#19 of ADA, the elastomeric impression materials must be able to reproduce in fine details of $25 \mathrm{im}$ or less ${ }^{3-6}$. The dimensional changes in elastomeric materials may occur due to many factors such as hydrophilicity, polymerization shrinkage, byproduct evaporation from polymerization reaction, shrinkage from 
thermal alteration, incomplete elastic recovery, time elapsed for impression pouring, mishandling, thickness and adhesion of the material to the tray ${ }^{2,3,7-9}$.

Polyvinyl siloxanes do not present byproduct formation after the polymerization reaction, which is expected to guarantee dimensional stability. It was shown that it remains dimensionally stable for up to 7 days and, also after the preparation of a first cast, it is possible to make a subsequent cast from the same mold ${ }^{4,8,10}$. However, the additional polymerization reaction involves linking a vinyl siloxane in the base material with a hydrogen siloxane via a platinum catalyst, where it is expected to occur some material shrinkage. Moreover, the reaction produces hydrogen, which is scavenged by platinum or palladium ${ }^{2,9,11}$. Therefore, mold distortion is inevitable and should be minimized by improving the impression techniques.

Polyvinyl siloxanes are found in different viscosities (from very low to very high viscosity materials), making it possible to use different impression techniques: regular onestep (single phase), putty wash one-step (simultaneous impression technique) and putty wash two-step (reline impression technique $)^{2,4,8,10,12-19}$. The single phase impression technique employs only a regular body monophase material, which is inserted into an individual tray. Both the simultaneous and the reline impression techniques use silicone materials of two different viscosities, but in the last technique handling of both materials is performed at different times. In this case, a first impression is made with heavybody material (putty viscosity). After its polymerization, the impression is removed from the mouth and a uniform relief of approximately 1 to $3 \mathrm{~mm}$ is made in order to obtain a space for the fluid-body material (wash viscosity). After that, the fluid polyvinyl siloxane material is handled, inserted into the relieved mold and over the prepared teeth, and the tray is repositioned in the mouth until the polymerization reaction is complete co,10,13-14,18-19. $^{\text {. }}$

The main disadvantages of the reline impression technique are the time spent to relieve the putty material, the greater amount of debris generated by the relieving procedure in the clinical environment and lack of adhesion between the heavybody putty and light-body wash materials ${ }^{7,20}$. In order to eliminate this step, without losing the quality of the final work, this study aimed to present a modification of the reline impression technique, which we will call as modified reline impression technique (MRIT), as well as to assess the dimensional changes of polyvinyl siloxane impressions obtained by the MRIT and compare it with the conventional reline impression technique (CRIT) at different storage times (immediately, 1 h, 2 days and 7 days).

\section{Material and methods}

\section{Matrix fabrication}

An acrylic resin matrix was made by a CAD-CAM system (Zirkonzahn, Gais, Italy) for obtaining the polyvinyl siloxane specimens. For this purpose, an acrylic resin tablet (Temp Premium, Zirkonzahn) with $95 \mathrm{~mm}$ diameter and 16 mm thick was milled. The Zirkonzahn Archiv software (Zirkonzahn) was used to design three abutments (A, B and C) similar to a canine tooth randomly arranged from each other. After acrylic resin tablet milling (Zirkonzahn Frasen), the cusp tips of the teeth were cut using a tungsten cutter (Edenta, São Paulo, SP, Brazil) to generate a flat surface. Next, a cross was carved in this region on each tooth with a thin grained cutting disc (KG Sorensen, São Paulo, SP, Brazil). Moreover, three acrylic resin spacers (Duralay, Polidental, Cotia, Brazil) were made in the resin matrix to allow polyvinyl siloxane material flow during the impressions and thereby avoid the presence of bubbles (Figure 1).

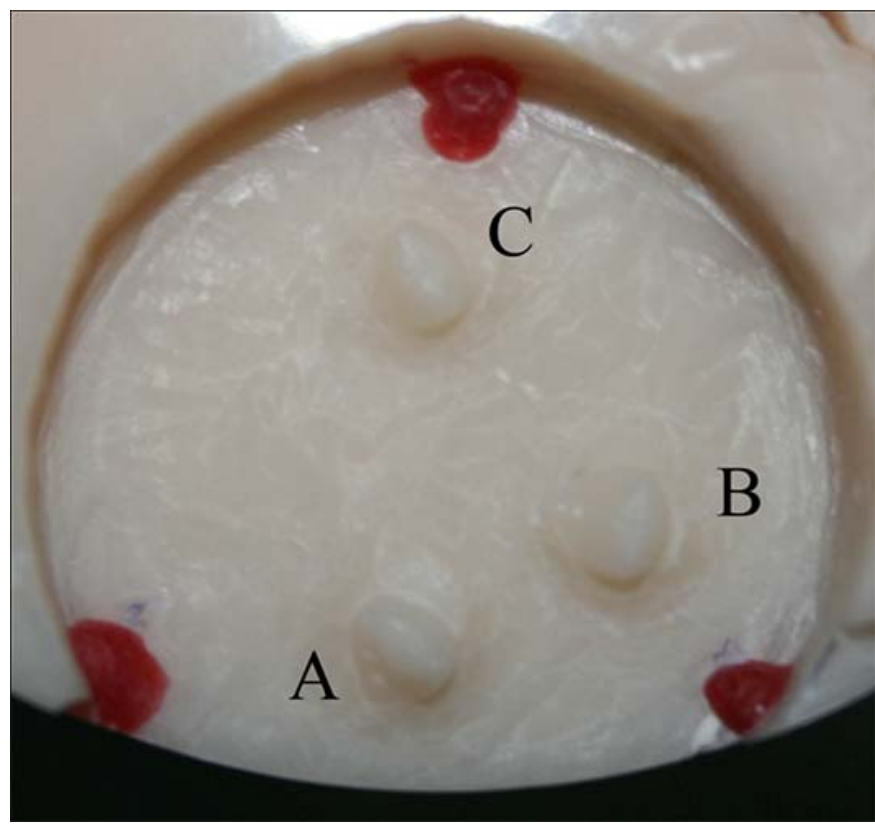

Fig. 1. Acrylic resin matrix used to obtain the addition silicone specimens, where it is possible to observe the three abutments $(A, B, C)$ similar to canine teeth that had their cusp tips sectioned.

\section{Polyvinyl siloxane specimen preparation}

In order to obtain the polyvinyl siloxane specimens (AD Futura, DFL, Rio de Janeiro, RJ, Brazil), the acrylic resin matrix was molded using both impression techniques $(n=5)$ : the conventional reline impression technique (CRIT) and the modified reline impression technique (MRIT). Round metallic devices (43 $\mathrm{mm}$ diameter and $5 \mathrm{~mm}$ high) were fabricated and used as trays.

\section{Conventional reline impression technique (CRIT)}

Initially, the high viscosity polyvinyl siloxane (AD Futura) was handled according to manufacturer's instructions, inserted into a metallic tray and positioned over the acrylic resin matrix. The set (tray/polyvinyl siloxane/acrylic resin matrix) was kept under pressure $(250 \mathrm{~kg})$ for $3 \mathrm{~min}$ by a hydraulic press (VH, Araraquara, SP,Brazil). Then, the impression was removed from the matrix and the relieving procedure was made using a tungsten cutter (Edenta, São Paulo, SP, Brazil), maintaining a uniform reduction of approximately $1.5 \mathrm{~mm}^{21}$. Next, the low viscosity polyvinyl 


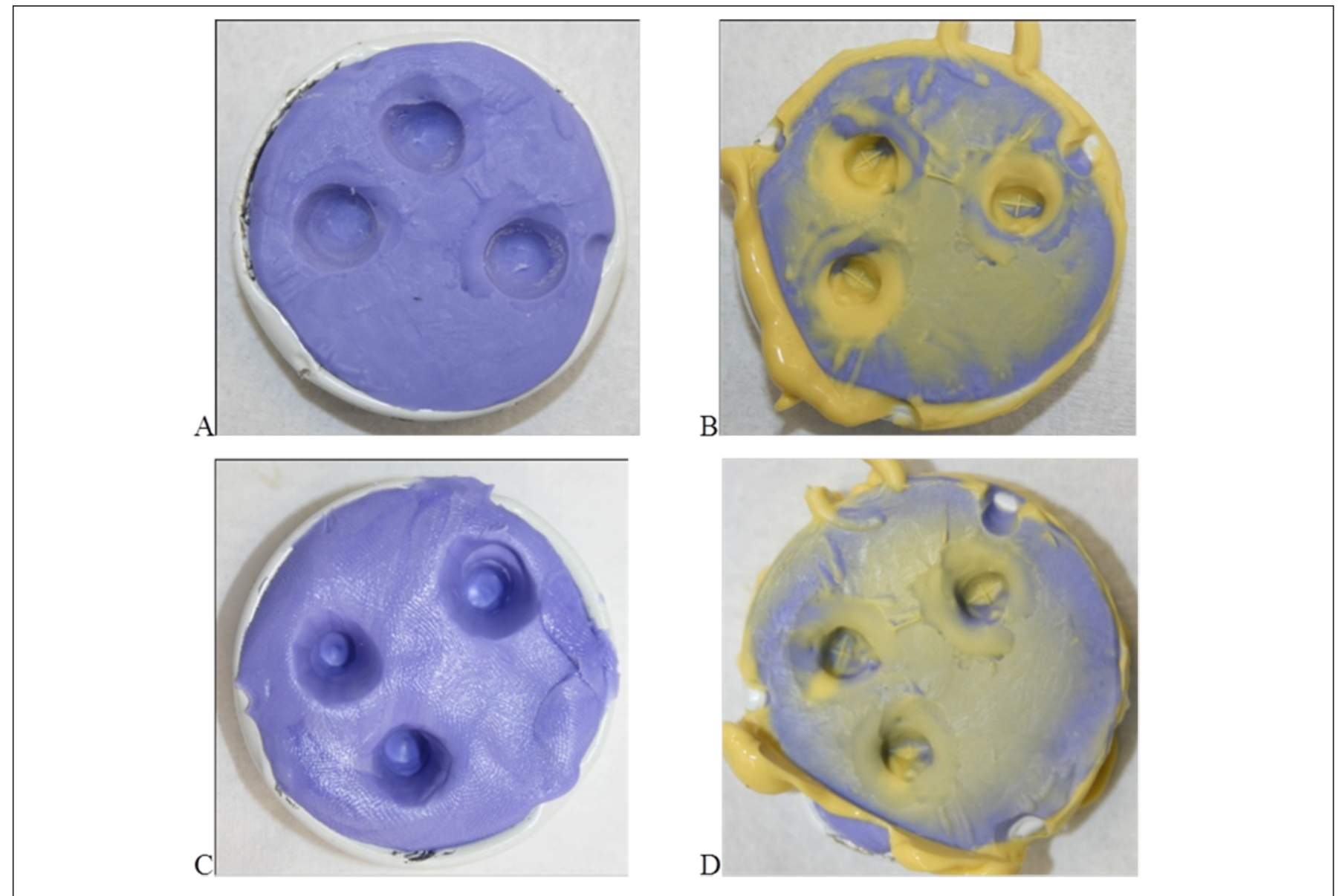

Fig. 2. $A$ and $B$ refer to the CRIT, where $A$ shows the impression relieving procedure with a tungsten cutter and $B$ shows the final impression. $C$ and $D$ refer to the MRIT, where $\mathrm{C}$ shows the impression relieving procedure by compression with a spatula handle and $\mathrm{D}$ shows the final impression.

siloxane (Futura AD) was inserted over the relieved impression with a self-mixing dispenser, repositioned over the matrix, and the set was taken to the hydraulic press $(250 \mathrm{~kg}, \mathrm{VH})$ for 3 min (Figure $2 \mathrm{~A}$ and $\mathrm{B}$ ).

\section{Modified reline impression technique (MRIT)}

The high viscosity polyvinyl siloxane (Futura) was handled according to the manufacturer's instructions, inserted into a metallic tray and positioned over the acrylic resin matrix. The set (tray/polyvinyl siloxane/acrylic resin matrix) was kept under pressure of $250 \mathrm{~kg}(\mathrm{VH})$. However, before completing the polyvinyl siloxane polymerization, the impression was separated from the matrix. Immediately, the impression's relieving procedure was made by compression using the handle of a \#36 dental cement spatula (Duflex SS White, Rio de Janeiro, RJ, Brazil). Even before the end of the polyvinyl siloxane polymerization, the impression was repositioned over the matrix and the end of its polymerization was awaited under a pressure of $250 \mathrm{~kg}$ for $3 \mathrm{~min}(\mathrm{VH})$. After that, the impression was removed from the matrix. Then, the low viscosity polyvinyl siloxane (Futura) was inserted over the relieved impression with a self-mixing dispenser, repositioned over the matrix, and the set was taken again to the hydraulic press $(250 \mathrm{~kg}, \mathrm{VH})$ for $3 \mathrm{~min}$ (Figure $2 \mathrm{C}$ and $\mathrm{D}$ ).

\section{Dimensional changes measurement of the polyviny siloxane impressions}

All impressions were stored dry at room temperature and the dimensional measurement changes were done at different storage times: immediately (I), $1 \mathrm{~h}(1 \mathrm{~h}), 2$ days $(2 \mathrm{~d})$ and 7 days (7d). Initially, the distances among the A, B and C abutments of the acrylic resin matrix were assessed using the Zirkonzahn Modellier software (Zirkonzahn). In order to decrease the superficial brightness of the matrix and allow the measurement of the distances, it was necessary to coat it with a white suspension solution (Spot Check Magnaflux SKD-S2, São Paulo, SP, Brazil). Thus, three measurements were taken as reference for evaluation of the impressions' dimensional changes: $\mathrm{AB}=12.896 \mathrm{~mm}, \mathrm{BC}=17.183 \mathrm{~mm}$ and $\mathrm{AC}=18.846 \mathrm{~mm}$. After that, the same distances $(\mathrm{AB}$, $\mathrm{BC}$ and $\mathrm{AC}$ ) in all polyvinyl siloxane impressions were obtained by the Zirkonzahn Modellier software at each storage time (Figure 3).

The differences between the impressions and their respective matrix reference measurements were calculated to determine the dimensional changes. Data for each distance ( $\mathrm{AB}, \mathrm{BC}$ and $\mathrm{AC})$ were subjected to one-way ANOVA. Tukey's test was used for multiple comparisons. Tests were performed with a pre-set significance level of $5 \%$. 


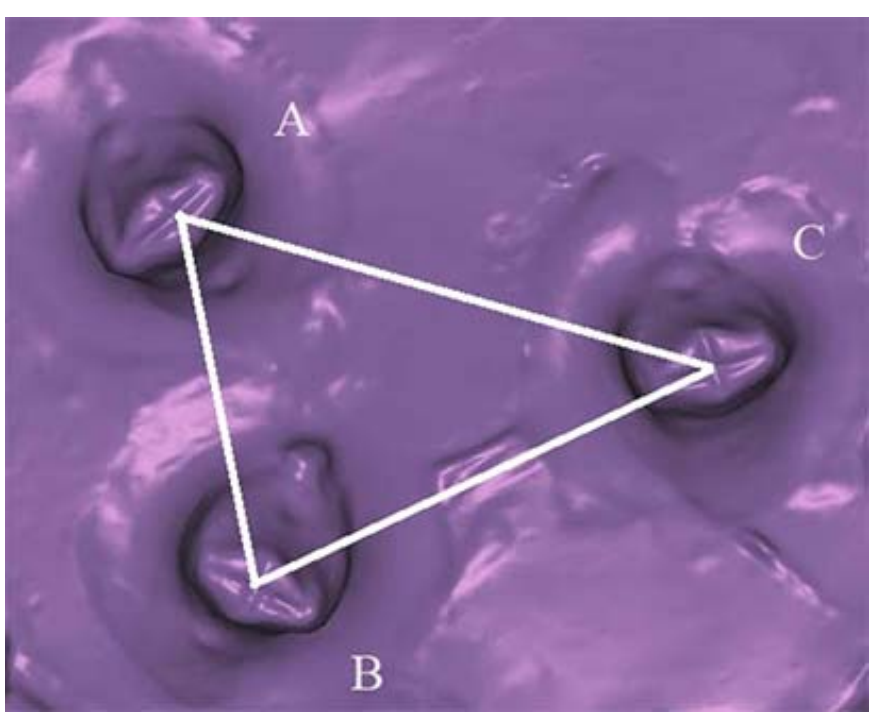

Fig. 3. ZirkonZhan Modellier software image of an addition silicone impression showing the measurement of the distances among the three abutments $(A B, B C$ and $\mathrm{AC}$ ).

\section{Results}

There was no statistically significant difference among the mean dimensional changes of the impressions made by both techniques, irrespective of the storage times, for $\mathrm{AB}$ $(\mathrm{p}=0.0597)$ and $\mathrm{BC}(\mathrm{p}=0.2167)$ distances. Tables 1 and 2 show the mean dimensional changes, standard deviations and confidence intervals (in $\mu \mathrm{m}$ ) of the experimental groups for $\mathrm{AB}$ and $\mathrm{BC}$ distances, respectively.

On the other hand, there was a statistically significant difference for dimensional change means of the experimental groups for the AC distance $(p=0.006)$. Table 3 and Figure 4 show the means, standard deviations and confidence intervals (in $\mu \mathrm{m}$ ) of dimensional changes of the tested experimental groups. For the immediate storage time, the means for both impression techniques were statistically different, in which CRIT impressions showed expansion, and MRIT impressions showed contraction in relation to the resin matrix dimensions. However, for other storage times, no statistically significant difference among the means of both impression techniques was found, but the same tendency of the CRIT impressions presenting expansion and MRIT impressions presenting contraction was observed.

\section{Discussion}

Factors such as non-simultaneous polymerization shrinkage of polyvinyl siloxane materials with different viscosities, the deficiency of detailed reproduction of the high viscosity impression material and high flow under pressure of the low viscosity material during the impression,

Table 1. Means, standard deviations and confidence intervals (in $\mu \mathrm{m}$ ) of dimensional changes for the AB distance of both impression techniques (CRIT and MRIT) according to the storage times: immediate (I), $1 \mathrm{~h}(1 \mathrm{~h}) 2$ days (2d) and 7 days $(7 \mathrm{~d}) . *$

\begin{tabular}{|c|c|c|c|c|c|}
\hline \multirow[t]{2}{*}{ Impression technique } & \multirow[t]{2}{*}{ Storage time } & \multirow[t]{2}{*}{ Mean } & \multirow[t]{2}{*}{ Standard deviation } & \multicolumn{2}{|c|}{ Confidence interval } \\
\hline & & & & Maximum & Minimum \\
\hline \multirow[t]{4}{*}{ CRIT } & I & 116.8 & 119.6 & 236.4 & -2.8 \\
\hline & $1 \mathrm{~h}$ & 38.8 & 151.1 & 189.9 & -112.3 \\
\hline & $2 d$ & 118.4 & 143.4 & 261.8 & -25.0 \\
\hline & $7 d$ & 76.8 & 73.8 & 150.6 & 3.0 \\
\hline \multirow[t]{4}{*}{ MRIT } & 1 & -42.2 & 142.8 & 100.6 & -185.0 \\
\hline & $1 \mathrm{~h}$ & -83.0 & 25.4 & -57.6 & -108.4 \\
\hline & $2 d$ & 71.0 & 69.2 & 140.2 & 1.8 \\
\hline & $7 d$ & -8.8 & 103.5 & 94.7 & -112.3 \\
\hline
\end{tabular}

* There was no statistically significant difference between the groups $(p=0.0597)$.

Table 2. Means, standard deviations and confidence intervals (in $\mu \mathrm{m}$ ) of dimensional changes for the $\mathrm{BC}$ distance of both impression techniques (CRIT and MRIT) according to the storage times: immediate (I), $1 \mathrm{~h} \mathrm{(1h)} 2$ days (2d) and 7 days $(7 \mathrm{~d})$. $^{*}$

\begin{tabular}{lccccc}
\hline Impression technique & Storage time & Mean & Standard deviation & \multicolumn{2}{c}{$\begin{array}{c}\text { Confidence interval } \\
\text { Minimum }\end{array}$} \\
CRIT & I & 98.8 & 156.1 & 254.9 & -57.3 \\
& 1h & 65.2 & 111.9 & 177.1 & -46.7 \\
& 2d & 95.8 & 118.6 & 214.4 & -22.8 \\
& 7d & 50.8 & 169.2 & 220.0 & -118.4 \\
\hline MRIT & I & -62.8 & 192.2 & 129.4 & -255.0 \\
& 1h & -49.2 & 68.9 & 19.7 & -118.1 \\
& 2d & -22.4 & 78.8 & 56.4 & -101.2 \\
& 7d & -55.6 & 73.2 & 17.6 & -128.8 \\
\hline
\end{tabular}

* There was no statistically significant difference between the experimental groups $(p=0.2167)$. 
Table 3. Means, standard deviations and confidence intervals (in $\mu \mathrm{m}$ ) of dimensional changes for AC distance of both impression techniques (CRIT and MRIT) according to the storage times: immediate (I), $1 \mathrm{~h}(1 \mathrm{~h}) 2$ days (2d) and 7 days $(7 \mathrm{~d})$.* $^{*}$

\begin{tabular}{|c|c|c|c|c|c|}
\hline \multirow[t]{2}{*}{ Impression technique } & \multirow[t]{2}{*}{ Storage time } & \multirow[t]{2}{*}{ Mean } & \multirow[t]{2}{*}{ Standard deviation } & \multicolumn{2}{|c|}{ Confidence interval } \\
\hline & & & & Maximum & Minimum \\
\hline \multirow[t]{4}{*}{ CRIT } & 1 & $195.2^{\mathrm{a}}$ & 161.5 & 236.4 & -2.8 \\
\hline & $1 \mathrm{~h}$ & $172.6^{\mathrm{ab}}$ & 200.7 & 189.9 & -112.3 \\
\hline & $2 d$ & $83.6^{\mathrm{ab}}$ & 126.1 & 261.8 & -25.0 \\
\hline & $7 d$ & $135.4^{\mathrm{ab}}$ & 143.8 & 150.6 & 3.0 \\
\hline \multirow[t]{4}{*}{ MRIT } & 1 & $-100.8^{b}$ & 141.1 & 100.6 & -185.0 \\
\hline & $1 \mathrm{~h}$ & $-75.2^{\mathrm{ab}}$ & 139.1 & -57.6 & -108.4 \\
\hline & $2 d$ & $-52.8^{a b}$ & 85.0 & 140.2 & 1.8 \\
\hline & $7 d$ & $-33.2^{\mathrm{ab}}$ & 96.5 & 94.7 & -112.3 \\
\hline
\end{tabular}

* Same letters indicate no statistically significant difference $(p>0.05)$.

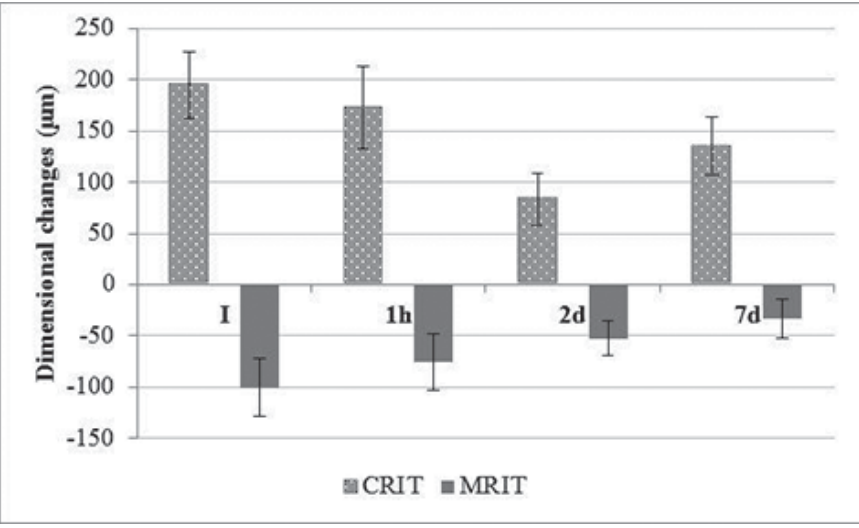

Fig. 4. Dimensional changes (in $\mu \mathrm{m}$ ) of the impression techniques (CRIT and MRIT) according to the storage times (immediately, $1 \mathrm{~h}, 2$ days and 7 days) for AC distance.

seem to explain the superiority of the reline impression technique to the simultaneous technique ${ }^{8,17-18}$. However, some disadvantages of the reline impression technique were reported, as the difficulty in repositioning the relieved impression in the mouth, the increase of clinical time and the amount of debris due to the impression's relieve procedure, which are commonly performed with a razor blade, drill or milling cutter $^{7,10}$. The modification of the conventional reline impression technique proposed in the present study eliminates such problems because the impression's relieving is performed by compression using the handle of a dental cement spatula and the repositioning is done before completing the polymerization of the material.

Several studies comparing different impression techniques found superior dimensional stability obtained by the conventional reline technique, which indicates that the impression's relieving procedure result in less distortion ${ }^{8,17-}$ ${ }^{18}$. Furthermore, it was demonstrated that this result was obtained both for $30 \mathrm{~s}$ after impression and 30 days of the impression's storage ${ }^{7}$. In this study, the impressions' dimensional changes obtained by the two techniques, in which both used some impression relieve procedure, was assessed immediately, $1 \mathrm{~h}, 2$ days and 7 days after the impression, which were the storage times previously reported as being able to identify possible dimensional changes of impression materials ${ }^{4,7-8,14}$. In both techniques and for most of storage times, except for the immediate time, the impressions maintained their dimensional stability, which is probably due to the fact that they were made with polyvinyl siloxane, were under constant pressure during the polymerization, presented a uniform thickness for the low viscosity polyvinyl siloxane, were free of bubbles and presented correct proportion between the base and catalyst pastes, due to the material handling with a self-mixing dispenser ${ }^{21}$.

In a previous study, the dimensional change of additional curing silicone and polyether was evaluated by plaster casts. Casts were made by multiple pouring at $30 \mathrm{~min}, 6 \mathrm{~h}, 24 \mathrm{~h}$ and 30 days after impression making. All deviations in casts made from silicone impression material were considered within a clinically acceptable range. On the other hand, for polyether the distortions were clinically unacceptable ${ }^{7}$. This result could be attributed to the good elastic recovery of the polyvinyl siloxanes, which corroborates the results of the present research (except for the immediate time for the AC distance) and other studies ${ }^{7,9}$. Besides the dimensional stability of polyvinyl siloxane, the elastic recovery is an essential property these materials must have to guarantee the impression's accuracy ${ }^{4}$. Elastic recovery of an impression is defined as the ability of a material to return to its original dimensions without significant distortion upon removal from the mouth ${ }^{3,9}$. The significant difference between the evaluated techniques for the immediate storage time for the $\mathrm{AC}$ distance may be the result of an incomplete elastic recovery of the impression material.

The measurements of the present study did not consider any tooth movement. If it were to consider the periodontal ligament space, which was previously reported to range from 90 to $240 \mu \mathrm{m}^{22}$, the dimensional changes found in the impressions of the present study, which ranged from 9 to $195 \mu \mathrm{m}$, could be compensated by periodontal movement and this material distortion could be considered clinically acceptable ${ }^{7}$.

Another important result observed in this study is that generally the CRIT promoted the polyvinyl siloxane expansion (positive values of dimensional changes), while the MRIT allowed the material shrinkage (negative values of dimensional changes). Considering that the polyvinyl 
siloxanes are polymers that naturally undergo polymerization shrinkage, a more coherent response occurred by MRIT.

Several studies evaluated the dimensional changes of impression materials by means of plaster casts and the measurements were carried out by different devices such as microscope, digital caliper, three-dimensional Zeiss meter and stereomicroscope $\mathrm{e}^{4,7,13-14,23}$. However, the measurements in the present study were performed directly in the impressions using the Zirkonzahn system, which ensured accuracy and practicality in impression evaluation, and allowed the visualization of three-dimensional images. Thus, this methodology excluded the possibility of changes in plaster casts and wrong positioning of the specimens during measurement. Based on that and according to ADA's specification \#19 and previous studies, five specimens of each experimental group are appropriate to perform linear dimensional change tests ${ }^{5,7,16,24}$. Moreover, the use of a CADCAM system to fabricate the acrylic resin matrix resulted in a master model with good mechanical resistance and surface finishing.

Moreover, considering that the impression is a timedependent procedure ${ }^{14}$, it is also recommended to wait at least $1 \mathrm{~h}$ to pour when it is made with a polyvinyl siloxane material. However, more experimental studies to verify detail reproduction and clinical studies are required for this new impression technique.

Based on the results of this study it was possible to conclude that: 1. For all distances and both impression techniques, there was no significant difference among the dimensional change means for $1 \mathrm{~h}, 2$ days and 7 days of storage time; 2. For the AC distance, there was a significant difference between the dimensional change means obtained by the CRIT and MRIT for immediate storage time; 3 . The MRIT may be recommended due to its simplicity, with a waiting time for pouring of at least $1 \mathrm{~h}$. Within the limitations of this research, it is feasible to suggest that the modification of the reline impression technique proposed in the present study is recommended because it is easy to carry out, reduces the time spent for putty material relieving and decreases the amount of debris generated by the relieving procedure.

\section{Acknowledgements}

The authors would like to thank Dr. Marcelo Filietaz of the Masterdent Prótese Odontológica Ltda, Brazil for the CAD-CAM system availability.

\section{References}

1. Giordano R. Issues in handling impression materials. Gen Dent. 2000; 48: 646-8.

2. Giordano R. Impression materials: basic properties. Gen Dent. 2000; 48: 510-2, 514, 516.

3. Hamalian TA, Nasr E, Chidiac JJ. Impression materials in fixed prosthodontics: influence of choice on clinical procedure. J Prosthodont. 2011; 20: 153-60.
4. Chen SY, Liang WM, Chen FN. Factors affecting the accuracy of elastomeric impression materials. J Dent. 2004; 32: 603-9.

5. ANSI/ADA: Specification n.19-Dental elastomeric impression materials Chicago: American Dental Association; 2004.

6. Tjan AH, Li T, Logan GI, Baum L. Marginal accuracy of complete crowns made from alternative casting alloys. J Prosthet Dent. 1991; 66: 157-64.

7. Thongthammachat S, Moore BK, Barco MT, Hovijitra S, Brown DT, Andres CJ. Dimensional accuracy of dental casts: influence of tray material, impression material, and time. J Prosthodont. 2002; 11: 98-108.

8. Al-Bakri IA, Hussey D, Al-Omari WM. The dimensional accuracy of four impression techniques with the use of addition silicone impression materials. J Clin Dent. 2007; 18: 29-33.

9. Papadogiannis D, Lakes R, Palaghias G, Papadogiannis $Y$. Effect of storage time on the viscoelastic properties of elastomeric impression materials. J Prosthodont Res, 2012; 56: 11-8.

10. Shiozawa M, Takahashi H, Iwasaki N. Effects of the space for wash materials on sulcus depth reproduction with addition-curing silicone using two-step putty-wash technique. Dent Mater J. 2013; 32: 150-5.

11. Derrien G, Le Menn G. Evaluation of detail reproduction for three die materials by using scanning electron microscopy and two-dimensional profilometry. J Prosthet Dent. 1995; 74: 1-7.

12. Christensen GJ. What category of impression material is best for your practice? J Am Dent Assoc. 1997; 128: 1026-8.

13. Franco EB, da Cunha LF, Herrera FS, Benetti AR. Accuracy of singlestep versus 2-step double-mix impression technique. ISRN Dent. 2011; 2011: 341-546.

14. Franco EB, da Cunha LF, Benetti AR. Effect of storage period on the accuracy of elastomeric impressions. J Appl Oral Sci. 2007; 15: 195-8.

15. Idris B, Houston F, Claffey N. Comparison of the dimensional accuracy of one- and two-step techniques with the use of putty/wash addition silicone impression materials. J Prosthet Dent. 1995; 74: 535-41.

16. Hung SH, Purk JH, Tira DE, Eick JD. Accuracy of one-step versus twostep putty wash addition silicone impression technique. J Prosthet Dent. 1992; 67: 583-9.

17. Caputi S, Varvara G. Dimensional accuracy of resultant casts made by a monophase, one-step and two-step, and a novel two-step putty/light-body impression technique: an in vitro study. J Prosthet Dent. 2008; 99: 274-81.

18. Nissan J, Rosner O, Bukhari MA, Ghelfan O, Pilo R. Effect of various putty-wash impression techniques on marginal fit of cast crowns. Int J Periodontics Restorative Dent. 2013; 33: e37-42.

19. Nissan J, Gross M, Shifman A, Assif D. Effect of wash bulk on the accuracy of polyvinyl siloxane putty-wash impressions. J Oral Rehabil. 2002; 29: 357-61.

20. Purk JH, Hung SH, Chappell RP, Casper RL, Eick JD. The effect of time on the adhesion of light-body to heavy-body Express in the two-step reline polyvinylsiloxane impression technique. Am J Dent. 1990; 3: 249-52.

21. Levartovsky S, Levy G, Brosh T, Harel N, Ganor Y, Pilo R. Dimensional stability of polyvinyl siloxane impression material reproducing the sulcular area. Dent Mater J. 32: 25-31.

22. Coolidge ED. The thickness of the human periodontal membrane. J Am Dent Assoc. 1937; 24: 1260-70.

23. Thielke S, Serrano JG, Lepe X. A method for true coordinate threedimensional measurement of casts using a measuring microscope. JProsthet Dent. 1998; 80: 506-10.

24. Klooster J, Logan GI, Tjan AH. Effects of strain rate on the behavior of elastomeric impression. J Prosthet Dent. 1991; 66: 292-8. 